\title{
Potts Models on Feynman Diagrams
}

\author{
D.A. Johnston \\ Dept. of Mathematics \\ Heriot-Watt University \\ Riccarton \\ Edinburgh, EH14 4AS, Scotland \\ and \\ P. Plecháč \\ Mathematical Institute \\ 24-29 St Giles' \\ Oxford \\ OX1 3LB
}

28 April 1997

\begin{abstract}
We investigate numerically and analytically Potts models on "thin" random graphs - generic Feynman diagrams, using the idea that such models may be expressed as the $N \rightarrow 1$ limit of a matrix model. The thin random graphs in this limit are locally tree-like, in distinction to the "fat" random graphs that appear in the planar Feynman diagram limit, $N \rightarrow \infty$, more familiar from discretized models of two dimensional gravity.

The interest of the thin graphs is that they give mean field theory behaviour for spin models living on them without infinite range interactions or the boundary problems of genuine tree-like structures such as the Bethe lattice. $q$-state Potts models display a first order transition in the mean field for $q>2$, so the thin graph Potts models provide a useful test case for exploring discontinuous transitions in mean field theories in which many quantities can be calculated explicitly in the saddle point approximation.

Such discontinuous transitions also appear in multiple Ising models on thin graphs and may have implications for the use of the replica trick in spin glass models on random graphs.
\end{abstract}




\section{Introduction and Reprise of Continuous Transitions}

A simple and elegant method of describing spin models on random graphs, drawing inspiration from the matrix model methods [1] used to describe planar random graphs in two-dimensional gravity, was first proposed in 2. It was observed that the requisite ensemble of random graphs of unrestricted topology could be thought of as arising from the perturbative Feynman diagram expansion of a scalar integral in much the same manner as the planar graphs that appear in two dimensional gravity theories were generated from the perturbative expansion of a matrix integral. In effect, the unrestricted random graphs appear in the $N \rightarrow 1$ limit of an $N \times N$ Hermitian matrix model, which we denote as "thin" graphs, to distinguish them from the planar "fat" graphs which appear in the $N \rightarrow \infty$ limit and still retain their matrix structure. Throughout the paper we will use "thin graphs" and "Feynman diagrams" interchangeably to denote the random graphs of unrestricted topology on which our spin models live. Spin models on random graphs are of interest as they will display mean field behaviour because the graphs have a tree-like local structure [3]. The advantage of using random graphs, which are closed, over genuine tree-like structures such as the Bethe lattice is that dominant boundary effects are absent. The complications, both analytical and numerical, of being forced to consider only sites deep within the lattice are thus absent. Other ways of accessing mean field behaviour, such as infinite range interactions, are not very well suited for numerical simulation.

In previous papers we showed that the thin graphs of the Feynman diagram expansion offered a practical method of investigating mean field models both analytically and numerically. The equilibrium behaviour of ferromagnetic Ising models [ [ 4 and spin glasses [5] was found to parallel that of the equivalent model on the appropriate Bethe lattice with the same number of neighbours [6], and the analytical treatment offered a different perspective to previous approaches to random graph spin models and spin glasses [7, 8]. The investigation of dynamical phenomena such as aging effects in spin glasses [9] was also found to be facilitated by random graph simulations. Other authors have also employed random graphs in simulations of the random field Ising model 10 in order to avoid boundary problems with the Bethe lattice.

Analytical calculations using the approach of [2] involve simple saddle point methods for standard integrals, or quantum mechanical path integrals in the case of continuous spins [11. If we consider undecorated random graphs, taking a $\phi^{3}$ theory for definiteness which will generate 3-regular random graphs 7, the number of such graphs with $2 n$ vertices can be calculated as

$$
N_{n}=\frac{1}{2 \pi i} \oint \frac{d \lambda}{\lambda^{2 n+1}} \int_{-\infty}^{\infty} \exp \left(-\frac{1}{2} \phi^{2}+\frac{\lambda}{6} \phi^{3}\right)
$$

which, when evaluated using a saddle point approximation, gives the correct counting

$$
N_{n}=\left(\frac{1}{6}\right)^{2 n} \frac{(6 n-1) ! !}{(2 n) ! !} .
$$

To include an Ising model we now decorate the vertices of the graphs with Ising spins having a Hamiltonian

$$
H=\beta \sum_{<i j>}\left(\sigma_{i} \sigma_{j}-1\right)
$$

where the sum is over nearest neighbour sites. The partition function is then given by

$$
Z_{n}(\beta) \times N_{n}=\frac{1}{2 \pi i} \oint \frac{d \lambda}{\lambda^{2 n+1}} \int \frac{d \phi_{+} d \phi_{-}}{2 \pi \sqrt{\operatorname{det} K}} \exp (-S),
$$

where $K$ is defined by

$$
K_{a b}^{-1}=\left(\begin{array}{cc}
1 & -c \\
-c & 1
\end{array}\right)
$$

\footnotetext{
${ }^{1}$ We will restrict ourselves to $\phi^{3}$ or 3-regular random graphs throughout. The saddle point equations may still be solved with larger numbers of neighbours, but become rapidly more complicated.
} 
and the action itself is a direct transcription of the matrix model action [12] to simple scalar variables

$$
S=\frac{1}{2} \sum_{a, b} \phi_{a} K_{a b}^{-1} \phi_{b}-\frac{\lambda}{3}\left(\phi_{+}^{3}+\phi_{-}^{3}\right) .
$$

The sum in the above runs over \pm indices 2 . The coupling $c=\exp (-2 \beta)$ and the $\phi_{+}$field can be thought of as representing "up" spins with the $\phi_{-}$field representing "down" spins. It is necessary to include the counting factor $N_{n}$ to disentangle the factorial growth of the undecorated graphs from any non-analyticity due to phase transitions in the decorating spins. One is also obliged to pick out the $2 n$-th order in the expansion explicitly with the contour integral over $\lambda$ as, unlike the planar graphs of two dimensional gravity, $\lambda$ cannot be tuned to a critical value to cause a divergence.

The mean field Ising transition manifests itself in this formalism as an exchange of dominant saddle points. Solving the saddle point equations $\partial S / \partial \phi_{ \pm}=0$

$$
\begin{aligned}
& \phi_{+}=\phi_{+}^{2}+c \phi_{-} \\
& \phi_{-}=\phi_{-}^{2}+c \phi_{+}
\end{aligned}
$$

(which we have rescaled to remove $\lambda$ and an irrelevant overall factor) we find a symmetric high temperature solution

$$
\phi_{+}=\phi_{-}=1-c
$$

which bifurcates at $c=1 / 3$ to the low temperature solutions

$$
\begin{aligned}
& \phi_{+}=\frac{1+c+\sqrt{1-2 c-3 c^{2}}}{2} \\
& \phi_{-}=\frac{1+c-\sqrt{1-2 c-3 c^{2}}}{2} .
\end{aligned}
$$

The bifurcation point is determined by the value of $c$ at which the high and low temperature solutions for $\phi$ are identical, which appears at the zero of the Hessian $\operatorname{det}\left(\partial^{2} S / \partial \phi^{2}\right)$. The magnetisation order parameter for the Ising model can also be transcribed directly from the matrix model 12

$$
M=\frac{\phi_{+}^{3}-\phi_{-}^{3}}{\phi_{+}^{3}+\phi_{-}^{3}}
$$

and shows a continuous transition with mean field critical exponent $(\beta=1 / 2)$. The other critical exponents may also be calculated and take on mean field values.

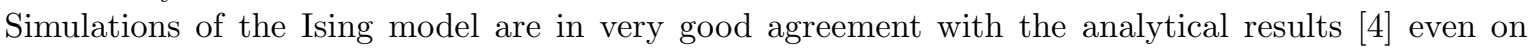
a single graph, which at first sight is rather surprising as the saddle point calculations are formally for an annealed ensemble of graphs. This appears to be true for all models where one might expect self-averaging, such as ferromagnetic Ising and Potts models - one large graph can be thought of as a collection of smaller graphs in these cases. With spin glasses it is still obligatory to consider a quenched ensemble of random graphs in order to take a (quenched) average over the disorder.

\section{Potts Models}

The Hamiltonian for a q-state Potts model can be written

$$
H=\beta \sum_{<i j>}\left(\delta_{\sigma_{i}, \sigma_{j}}-1\right)
$$

where the spins $\sigma_{i}$ take on $q$ values. The matrix model actions for such Potts models are well known (though only solved exactly so far for $q=3$ [13]). For the 3 -state Potts model the action is

$$
S=\frac{1}{2}\left(\phi_{1}^{2}+\phi_{2}^{2}+\phi_{3}^{2}\right)-c\left(\phi_{1} \phi_{2}+\phi_{1} \phi_{3}+\phi_{2} \phi_{3}\right)-\frac{1}{3}\left(\phi_{1}^{3}+\phi_{2}^{3}+\phi_{3}^{3}\right),
$$

\footnotetext{
${ }^{2}$ We have rescaled the $\phi$ 's with respect to 4,6 , 9 for uniformity with the Potts model notation.
} 
which can be used as the action on thin graphs if one takes, as in the Ising case, the $\phi$ 's to be scalar variables. For a q-state Potts model $c=1 /(\exp (2 \beta)+q-2)$.

"Ising-like" solutions to the 3 and 4-state Potts models were presented in [5], the 3 state case being

$$
\begin{array}{rlrl}
\phi_{1,2,3} & =1-2 c & & (\text { High }) \\
\phi_{1,2} & =\frac{1+\sqrt{1-4 c-4 c^{2}}}{2} & & (\text { Low }), \\
\phi_{3} & =\frac{1+2 c-\sqrt{1-4 c-4 c^{2}}}{2} &
\end{array}
$$

These high temperature and low temperature solutions are equal at the zero of the Hessian $c=1 / 5$, i.e. $g=4$. Similarly, the 4-state Potts model has the action

$$
S=\frac{1}{2}\left(\phi_{1}^{2}+\phi_{2}^{2}+\phi_{3}^{2}+\phi_{4}^{2}\right)-c\left(\phi_{1} \phi_{2}+\phi_{1} \phi_{3}+\phi_{1} \phi_{4}+\phi_{2} \phi_{3}+\phi_{2} \phi_{4}+\phi_{3} \phi_{4}\right)-\frac{1}{3}\left(\phi_{1}^{3}+\phi_{2}^{3}+\phi_{3}^{3}+\phi_{4}^{3}\right),
$$

and solving the saddle point equations again gave Ising-like solutions

$$
\begin{array}{rlr}
\phi_{1,2,3,4} & =1-3 c & (\text { HighT }) \\
\phi_{1,2,3} & =\frac{1-c+\sqrt{1-6 c-3 c^{2}}}{2} & (\text { Low }), \\
\phi_{4} & =\frac{1+3 c-\sqrt{1-6 c-3 c^{2}}}{2} &
\end{array}
$$

where the solutions matched at $c=1 / 7, g=5$. The picture is repeated for higher $\mathrm{q}$, where the action is

$$
S=\frac{1}{2} \sum_{i=1}^{q} \phi_{i}^{2}-c \sum_{i<j} \phi_{i} \phi_{j}-\frac{1}{3} \sum_{i=1}^{q} \phi_{i}^{3}
$$

and one finds a high temperature solution of the form $\phi_{i}=1-(q-1) c, \forall i$ bifurcating to a broken symmetry solution $\phi_{i}=\ldots \phi_{q-1} \neq \phi_{q}$ at $g=q+1$.

These results are somewhat surprising on two counts. Firstly, the motivation for using thin graphs was that they provided easy access to mean field results. However, it is known that the mean field theory for Potts models predicts a first order transition for $q>2$. Secondly, all the thin graph results so far for various models have been identical to the corresponding Bethe lattices, even down to non-universal features like the transition temperatures. Explicit calculations on the Bethe lattice have also given first order behaviour for $q>2$ [14 and shown the values of $g$ obtained above for the 3 and 4 state models correspond to spinodal points on the Bethe lattice. The models hit a first order transition before attaining these points. One might therefore expect that a first order transition should be lurking in the saddle point solutions for the actions above, given the previous tendency for the thin graph results to slavishly parallel the Bethe lattice.

The resolution of the conundrum is implicit already in the solutions in equs.(13, 15) and their higher $q$ equivalents. If we look at the Ising solution of equ.(9) we can see that the low temperature branches become real exactly at the transition point, whereas the square roots in the $q>2$ Potts solutions become real at larger c, and hence higher temperature. The topology of the phase diagram is perhaps best understood by plotting the magnetisation against c, which we do in Fig.1 for the Ising solution and in Fig.2 for the 4 state Potts model (all other $q>2$ state model s being of similar form). Low temperature corresponds to small $c$ and high temperatures to large $c$ for all the Ising and Potts models. For conformity with the Potts notation we define a Potts style magnetisation for the Ising model as

$$
m=\frac{\phi_{+}^{3}}{\left(\phi_{+}^{3}+\phi_{-}^{3}\right)}, \frac{\phi_{-}^{3}}{\left(\phi_{+}^{3}+\phi_{-}^{3}\right)}
$$

\footnotetext{
3 The right hand side of the LowT solutions may be exchanged, as one might have expected on symmetry grounds, and we have used this freedom to put the solutions in a tidier form than in 5 .
} 
where the first variant gives the upper (solid) branch of the low temperature magnetisation curve in Fig.1 and the second the lower (dashed) branch. Both definitions give the same value for the horizontal (dotted) high temperature solution with $m=1 / 2$ as $\phi_{+}=\phi_{-}$there. We can see that the paramagnetic high temperature solution bifurcates at $\mathbf{O}(\mathrm{c}=1 / 3)$ to give the upper and lower magnetised low temperature branches.

The equivalent magnetisation $m$ for the Potts models is defined as

$$
m=\frac{\phi_{q}^{3}}{\left(\sum_{i=1}^{q} \phi_{i}^{3}\right)}
$$

on the upper low temperature branch where it gives a maximum value and

$$
m=\frac{\phi_{1}^{3}}{\left(\sum_{i=1}^{q} \phi_{i}^{3}\right)}
$$

on the lower low temperature branch, where it gives a minimum. On the symmetric high temperature branch (dotted) both the definitions are, of course, equivalent and one finds $m=1 / q$. The standard Potts model order parameter can then be defined as

$$
M=\frac{q \max (m)-1}{q-1} .
$$

which is zero in the high temperature paramagnetic phase and tends to one in the magnetised low temperature phase.

The solutions of equs.(15) give the lower (dashed) branch in Fig.2, descending from the point $\mathbf{O}$ at which the square roots become real. We can see that the magnetisation "pitchfork" of the Ising diagram becomes skewed for $q=4$ (and all other $q>2$ ). The most important feature is that the upper (solid) branch does not connect continuously with the (dotted) high temperature solution, which joins the lower branch at $\mathbf{P}$. The upper branch is simply obtained for all $q$ by choosing the opposite sign for the square roots to the lower branch. As can be seen in Fig.2 it corresponds to the true low temperature magnetised phase where $m \rightarrow 1$ (and hence $M \rightarrow 1$ ) as $T \rightarrow 0$. The first order transition, denoted by a vertical line with ends labelled $\mathbf{Q}$ in Fig.2 takes place when the free energy of the upper branch is equal to the free energy of the high temperature solution. In the saddle point approximation the free energy to lowest order in the number of vertices $n$ is just the action $S$ so the first order transition point is given by the $c$ value, and hence temperature satisfying

$$
\text { S(upper branch })=S(\text { high temperature }) .
$$

As one can see in Fig.2 as the temperature (i.e. c) is reduced a first order transition intervenes between the $\mathbf{Q}$ 's before $\mathbf{P}$ is reached. Similarly, as the temperature is increased from zero along the upper branch a first order transition occurs before $\mathbf{O}$ is reached. The portions of the magnetisation curve $\mathbf{P O}$ and the dotted horizontal line to the left of $\mathbf{P}$ represent unstable states, whereas $\mathbf{P Q}, \mathbf{Q O}$ and the lower dashed branch to the left of $\mathbf{P}$ represent meta-stable states 1 . The meta-stable portions of the curve would be accessible by superheating out of the magnetised phase $(\mathbf{Q O})$ or supercooling from the paramagnetic phase ( $\mathbf{Q P}, \mathbf{P} \rightarrow$ origin). For completeness, we have listed the stable low temperature solutions for $q=3,4,5,6$ state Potts models in Appendix.A, which are the $q$ values simulated in the next section.

Various features of the solutions merit discussion. Equ.(21) can be solved analytically for moderate $q$ values on $\phi^{3}$ graphs without too much difficulty, and all the solutions fit the following compact formula for the critical value of $c$ at $\mathbf{Q}$

$$
c(Q)=\frac{1-(q-1)^{-1 / 3}}{q-2} .
$$

Indeed, if one takes the conjectured $q$ state solutions in Appendix.A it is possible to write down the saddle point action on the (upper) low temperature branch in terms of $\phi=\phi_{1 \ldots q-1}$ and $\tilde{\phi}=\phi_{q}$ by substituting

\footnotetext{
${ }^{4}$ These considerations, and indeed the two figures, are essentially identical to those for the Bethe lattice in [14].
} 
them into equ. 16)

$$
S=\frac{1}{2}(q-1)[1-c(q-2)] \phi^{2}-\frac{1}{3}(q-1) \phi^{3}+\frac{1}{2} \tilde{\phi}^{2}-\frac{1}{3} \tilde{\phi}^{3}-c(q-1) \phi \tilde{\phi}
$$

and similarly on the high temperature branch

$$
S=\frac{q}{2}(1-c(q-1)) \phi_{0}^{2}-\frac{q}{3} \phi_{0}^{3}
$$

where $\phi_{0}=1-(q-1) c$. Setting (equ.(23)) and (equ.(24)) equal, one also obtains the same $c(Q)$ as in equ. (22) above.

It is possible to calculate the jump in the magnetisation $\Delta M$ along the vertical line at $\mathbf{Q}$ and one finds in all cases

$$
\Delta M=\frac{q-2}{q-1} .
$$

If one now refers back to the Bethe lattice calculations of [14] one can see that $\Delta M$ is identical to that observed on the Bethe lattice. In addition, allowing for the differences in conventions ?, the formulae for the critical coupling $c(P)$ in equ.(22) is also identical to that for the Bethe lattice transition. The zero of the Hessian for the $q$ state Potts model action gives us the value of $c$ at $\mathbf{P}$ where the high temperature solution joins the lower branch

$$
c(P)=\frac{1}{2 q-1}
$$

and if we assume that the conjectured $q$ state low temperature solution in Appendix.A is correct, we can also calculate the value of $c$ at $\mathbf{O}$ where the square roots become real

$$
c(O)=\frac{q-1-2 \sqrt{q-1}}{(q-1)(q-5)}
$$

( $q=5$ can be handled by taking the limit $q \rightarrow 5$ ). As $q$ is increased the separation between points points $\mathbf{O}$ and $\mathbf{P}$ increases. In Table.1 below we list for convenience the $c$ values of points $\mathbf{O}, \mathbf{P}$ and $\mathbf{Q}$ for the $q=3,4,5,6$ state Potts models.

\begin{tabular}{|c|c|c|c|}
\hline$q$ & $c(O)$ & $c(P)$ & $c(Q)$ \\
\hline 3 & 0.20711 & 0.20000 & 0.20630 \\
\hline 4 & 0.15470 & 0.14286 & 0.15332 \\
\hline 5 & 0.125 & 0.11111 & 0.12335 \\
\hline 6 & 0.10557 & 0.09091 & 0.10380 \\
\hline
\end{tabular}

Table 1: The $c$ values for the points $\mathbf{O}, \mathbf{P}, \mathbf{Q}$ along with $\beta_{\text {crit }}$ for $q=3,4,5,6$ state Potts models The values of $c(O)$ and $c(P)$ that we have found are again identical to those on the Bethe lattice.

\section{Simulations}

The acid test of the saddle point solutions is whether they match up with simulations. We do not attempt a high accuracy verification of the analytical results here, but rather a consistency check on the first order nature of the transition and a verification of the values for $c(Q)$ and $\Delta M$ calculated in the previous section. To this end we generated single $\phi^{3}$ graphs with 250, 1000 and 2500 vertices for each of the $q$ state models. We verified the results by repeating all the simulations on a second, different graph for each size with identical results within the error bars in all cases.

\footnotetext{
${ }^{5}$ The $\theta$ of 14 is equal to $\exp (-2 \beta)$ in our notation.
} 
The generation of the graphs is easier than the planar $\phi^{3}$ graphs used in $2 d$ gravity simulations because of the absence of a constraint on the topology. This obviates the need to use (for instance) Tutte's algorithm in producing the graphs. The simulation itself used the Wolff algorithm, which will not beat the super-exponential slowing down right at the first order transition point, but is expected to be efficient elsewhere. We simulated a range of $\beta$ values, allowing 20,000 equilibration sweeps followed by $20,000 \times N$ cluster updates, where $N$ was $O(10)$ - the exact value depending on the mean cluster size. Measurements were made every $N$ cluster updates of all the standard thermodynamic quantities, the energy $E$, the magnetisation $M$, specific heat $C$ and magnetic susceptibility $\chi$. We also measured various Binder's cumulants for the magnetisation $\left\langle M^{4}>/<M^{2}\right\rangle^{2}$ and energy $\left.\left.<E^{4}\right\rangle /<E^{2}\right\rangle^{2}$ as well as correlation functions and autocorrelations.

We focus our attention first on the magnetisation curves for the various models, which are presented, with fortuitous numbering, in Figs.3,4 for the $q=3,4$ state models on graphs with 250,1000 and 2500 vertices. The smaller graphs display greater finite size rounding, but by the time one has got to 2500 vertices the agreement with the magnetisation calculated from the saddle point solutions (which are formally for an infinite number of vertices) is already quite good. On both plots we have delineated the expected critical points and jumps in the magnetisations. This agreement deteriorates somewhat for a given lattice size as $q$ and hence the strength of the transition increases. The $\beta_{\text {crit }}$ for various $q$ are estimated from the simulations by looking at the crossing of Binder's magnetisation cumulant $<M^{4}>/<M^{2}>^{2}$ for the various graph sizes. As one can see in Fig.5 for the 3 state Potts model (which is representative) the errors in the measurement of the cumulant are quite large, but even given this the estimated critical temperatures are all close to those calculated in the saddle point approximation. We also list the $\beta$ values for the two spinodal points $\mathbf{O}$ and $\mathbf{P}$ for comparison. As one can see even the rather modest simulations carried out here are sufficient to show that the spinodal point $\mathbf{P}$ can be excluded as the transition point in all cases. The results for all but the 3 state model cannot definitively exclude the other spinodal point $\mathbf{O}$ as the critical point, but the first order nature of the transition and the value of the jump in the magnetisation, as discussed below, favour a transition at $\mathbf{Q}$ as predicted by the saddle point calculations.

\begin{tabular}{|c|c|c|c|c|}
\hline$q$ & 3 & 4 & 5 & 6 \\
\hline$\beta_{\text {crit }}$ & $0.674(2)$ & $0.75(1)$ & $0.81(1)$ & $0.87(1)$ \\
\hline$\beta(Q)$ & 0.67369 & 0.75451 & 0.81533 & 0.86441 \\
\hline \hline$\beta(O)$ & 0.67122 & 0.74804 & 0.80472 & 0.84986 \\
\hline$\beta(P)$ & 0.69315 & 0.80470 & 0.89588 & 0.97295 \\
\hline
\end{tabular}

Table 2: The estimated $\beta_{\text {crit }}$, along with the calculated $\beta(Q), \beta(O), \beta(P)$.

The $\Delta M$ values are estimated by eye-balling the magnetisation curves for largest graphs and are consequently to be taken with a larger pinch of salt than the other measurements, but they are all consistent with the $(q-2) /(q-1)$ calculated in the previous section. We tabulate the results for the magnetisation jumps measured from the simulations below in Table.3 along with $(q-2) /(q-1)$ for comparison.

\begin{tabular}{|c|c|c|c|c|}
\hline$q$ & 3 & 4 & 5 & 6 \\
\hline$\Delta M$ & $0.50(5)$ & $0.68(1)$ & $0.72(5)$ & $0.9(2)$ \\
\hline$(q-2) /(q-1)$ & 0.5 & 0.66667 & 0.75 & 0.8 \\
\hline
\end{tabular}

Table 3: The estimated $\Delta M$ along with the calculated $(q-2) /(q-1)$.

Further confirmation that the transitions are indeed first order can be obtained by looking at the values of the Binder's energy cumulant near $\beta_{\text {crit }}$. This is expected to scale to $2 / 3$ for a continuous 
transition and a value less than $2 / 3$ if the transition is first order. The values are listed in Table. 4 and show a clear tendency to decrease around $\beta_{\text {crit }}$ that grows stronger with increasing $q$.

\begin{tabular}{|c|c|c|c|c|}
\hline$q$ & 3 & 4 & 5 & 6 \\
\hline$\frac{<E^{4}>}{\left.<E^{2}\right\rangle^{2}}$ & 0.664 & 0.65 & 0.64 & 0.60 \\
\hline
\end{tabular}

Table 4: The values of Binder's energy cumulant at the estimated $\beta_{\text {crit }}$

The energy itself is calculable from $E=-\partial \log Z / \partial \beta$ and is discontinuous at a first order transition. One finds values that are again in good agreement with the simulations by the time one has reached 2500 vertices. The measurements for the 4 state Potts model are shown in Fig.6. In a similar vein quantities such as the specific heat $C=\beta^{2}\left(\partial^{2} \log Z / \partial \beta^{2}\right)$ or the magnetic susceptibility may be calculated from the saddle point solutions and all give very satisfactory agreement with the measured quantities in the simulations. We do not describe these here as it is clear from the results already presented that, even given the limitations of the fairly modest simulations, there is ample support for the correctness of the saddle point solutions and the picture of the first order transition that they suggest.

In closing, we note that something akin to a standard finite size scaling analysis is possible with the thin graph approach to simulations, as witnessed by the use of the Binder's cumulant to estimate the critical temperature in the current work. The place of the factor $L^{-1 / \nu}$ that appears in finite size scaling on standard lattices, where $L$ is the linear size of the lattice, is taken by $n^{-1 / \nu d}$ where $n$ is the number of vertices in the graph. Although $d$ is formally infinite, the combination $\nu d$ is still well defined and all the scaling relations may be written in terms of this. Entirely analogous tactics have been used in the analysis of simulations of spin models in planar diagrams in theories of two-dimensional gravity, where $d$ in this case was a dynamically generated fractal dimension that was a priori unknown. On the analytical side $1 / n$ corrections may be obtained (and have been obtained already for the Ising model in [4]) by calculating the determinantal corrections to the saddle point solutions, so corrections to scaling can be obtained. Such issues would be worth pursuing if very high accuracy verification of the correspondence between calculation and simulation were required.

\section{Discussion}

Our previous analytical and numerical work [4, 5, 9] on spin models on Feynman diagrams had concentrated on the case of continuous transitions. The results in the current paper show that the simple saddle point equations that determine the phase structure of such models are also adequate to describe first order transitions. In the continuous case the critical point was pinpointed by finding the zeroes of the Hessian and corresponded to a bifurcation of magnetised states from the unmagnetised high temperature solution. This gives one the lower temperature spinodal point $\mathbf{P}$ in the Potts models, the true first order transition point at $\mathbf{Q}$ being determined by matching the saddle point actions (i.e. free energies) on the two branches of the solution. The upper spinodal point $\mathbf{O}$ is fixed as the point at which a square root appearing in the low temperature branches becomes real.

The critical temperatures we have calculated, the jump in the magnetisation and the magnetisation curves themselves are identical to the results obtained in [14] on the Bethe lattice. We thus conclude that the (mostly large) loops that are present in the Feynman graphs have no effect of the critical behaviour of ferromagnetic Potts models by comparison with the corresponding Bethe lattices. This is consistent with the earlier work on continuous transition which also demonstrated Bethe-lattice-like results. The loops will, however, have an effect in the antiferromagnetic models considered in [14], where a two-step invariant measure which presupposes a bipartite lattice was instrumental in the solution. As already noted in [4 for the antiferromagnetic Ising model, loops of both even and odd length are present in the Feynman diagrams, so frustration will be present on $\phi^{z}$ graphs if $q<z$. This apparently leads to a spin glass phase rather than antiferromagnetic order. In matrix models it is possible to arrange only even sided polygonations by using complex rather than Hermitian matrices, but it is not clear to us how to perform a similar trick on generic - rather than planar - Feynman diagrams. 
We find it rather remarkable that it is possible to write down the actions in equs. 23,24) for both the high and low temperature branches for arbitrary $q$, based on the ansatz for the $q$-state solutions in the Appendix. This is on a par with the results in [5] for the Hessian of an arbitrary number of Ising replicas on thin graphs. The availability of a solution where $q$ appears explicitly as a parameter opens the possibility of exploring the $q \rightarrow 1$ limit of the model, related to percolation, which we will address in a further publication. The percolative transition on the Bethe lattice has some unusual features [15], which one might also expect to manifest themselves on thin graphs. The behaviour of the model in an external field, considered in some detail on the Bethe lattice in [14], can also be investigated with the thin graph formalism both analytically and numerically without much by way of complications over the results presented here.

First order transitions also appear in the so-called Ising replica magnet [16], the finite $k$ version of the $k$-Ising replicas used in taking the $k \rightarrow 0$ limit ("replica trick") with quenched disorder. In these models on thin graphs the spin glass transition temperature appears as a sort of spinodal point [5] for all $k>2$, with the transition being continuous for $k=2$. A first order (in the overlap) transition intervenes before the putative continuous spin glass transition is reached for $k>2$. If it were not for this one would be tempted to argue that the $k \rightarrow 0$ limit for the spin glass transition temperature was trivial, as it is the same for all $k$. The role of the first order transition to a replica symmetric state when $k>2$ and the nature of the $k \rightarrow 0$ limit for thin graph models thus requires further elucidation. It is possible that the Potts models results presented here may cast some light on its properties.

The numerical work in this paper was intended as a consistency check of the formalism, rather than a full scale numerical investigation and finite size scaling analysis of the models. Nonetheless, it is clear from the results presented that the transitions are first order as predicted. The agreement between the simulations and the saddle point calculations for the critical temperatures and the observed jumps in the magnetisations are very satisfactory even on graphs with 2500 vertices.

In summary, we have seen that the thin graph approach is a convenient way of performing calculations and simulations for mean field Potts models with first order phase transitions. Such models, as well as being of interest in their own right, may help in understanding mean field spin glass and percolative transitions. 


\section{Appendix A}

We list here the low temperature solutions (upper branch in Fig.2) for the $q=3,4,5,6$ state Potts models which are referred to in the text. Note that the solutions appear follow a regular pattern and we have indicated the conjectured $q$ state solution (which fits the cases listed below, the Ising model $q=2$, and all the other higher $q$ solutions we checked explicitly before exhaustion set in) at the end. Just as for the lower branch solutions where the signs in front of the square roots are reversed, the right hand sides of the solutions can be exchanged for a given $q$.

\section{3-State}

$$
\begin{aligned}
\phi_{1,2} & =\frac{1-\sqrt{1-4 c-4 c^{2}}}{2} \\
\phi_{3} & =\frac{1+2 c+\sqrt{1-4 c-4 c^{2}}}{2} .
\end{aligned}
$$

\section{4-State}

$$
\begin{aligned}
\phi_{1,2,3} & =\frac{1-c-\sqrt{1-6 c-3 c^{2}}}{2} \\
\phi_{4} & =\frac{1+3 c+\sqrt{1-6 c-3 c^{2}}}{2}
\end{aligned}
$$

\section{5-State}

$$
\begin{aligned}
\phi_{1,2,3,4} & =\frac{1-2 c-\sqrt{1-8 c}}{2} \\
\phi_{5} & =\frac{1+4 c+\sqrt{1-8 c}}{2}
\end{aligned}
$$

\section{6-State}

$$
\begin{aligned}
\phi_{1,2,3,4,5} & =\frac{1-3 c-\sqrt{5 c^{2}-10 c+1}}{2} \\
\phi_{6} & =\frac{1+5 c+\sqrt{5 c^{2}-10 c+1}}{2}
\end{aligned}
$$

q-State

$$
\begin{aligned}
\phi_{1 \ldots q-1} & =\frac{1-(q-3) c-\sqrt{1-2(q-1) c+(q-5)(q-1) c^{2}}}{2} \\
\phi_{q} & =\frac{1+(q-1) c+\sqrt{1-2(q-1) c+(q-5)(q-1) c^{2}}}{2}
\end{aligned}
$$




\section{References}

[1] For a review see, J. Ambjorn, "Quantisation of Geometry" Les Houches 1994, hep-th/9411179.

[2] C. Bachas, C. de Calan and P. Petropoulos, J. Phys. A27 (1994) 6121.

[3] B. Bollobás, "Random Graphs", Academic Press, 1985.

[4] C. Baillie, D. Johnston and J-P. Kownacki, Nucl. Phys. B432 (1994) 551.

[5] C. Baillie, W. Janke, D. Johnston and P. Plecháč, Nucl. Phys. B450 (1995) 730;

C. Baillie and D. Johnston, Nucl. Phys. B47 (Proc. Suppl.) (1996) 649.

[6] H. A. Bethe, Proc. Roy. Soc. A 150 (1935) 552;

C. Domb, Advan. Phys. 9 (1960) 145;

T. P. Eggarter, Phys. Rev. B9 (1974) 2989;

E. Muller-Hartmann and J. Zittartz, Phys. Rev. Lett. 33 (1974) 893.

[7] L. Viana and A. Bray, J. Phys. C 18 (1985) 3037.

M. Mezard and G. Parisi, Europhys. Lett. 3 (1987) 1067;

I. Kanter and H. Sompolinsky, Phys. Rev. Lett. 58 (1987) 164;

K Wong and D. Sherrington, J. Phys. A20 (1987) L793;

K Wong and D. Sherrington, J. Phys. A21 (1988) L459;

C. de Dominicis and Y. Goldschmidt, J. Phys. A22 (1989) L775;

C. de Dominicis and Y. Goldschmidt, Phys. Rev. B41 (1990) 2184;

P-Y Lai and Y. Goldschmidt, J. Phys. A23 (1990) 399.

[8] S. Inawashiro and S. Katsura, Physica 100A (1980) 24;

S. Katsura, S. Inawashiro and S. Fujiki, Physica 99A (1979) 193;

S. Katsura, Physica 104A (1980) 333, ibid 141A (1987)556;

S. Katsura and S. Fujiki, J. Phys. C12 (1979) 1087;

D. Thouless, Phys. Rev. Lett. 56 (1986) 1082;

J. Chayes, L. Chayes, P Sethna and D. Thouless, Comm. Math Phys. 106 (1986) 41;

P. Mottishaw, Europhys. Lett. 4 (1987) 333;

K Wong and D. Sherrington, J. Phys. A20 (1987) L785.

[9] C. Baillie, D. Johnston, E. Marinari and C. Naitza, J. Phys. A29 (1996) 6683

[10] D. Dar, P. Shukla and J. Sethna, "Zero temperature hysteresis in the random field Ising model on a Bethe lattice", cond-mat/9611028.

[11] C. Baillie, N. Dorey, W. Janke and D. Johnston, Phys. Lett B369 (1996) 123.

[12] V. A. Kazakov, Phys. Lett. A119 (1986) 140;

D.V. Boulatov and V.A. Kazakov, Phys. Lett. B186 (1987) 379;

Z. Burda and J. Jurkiewicz, Acta Physica Polonica B20 (1989) 949.

[13] J-M. Daul, "Q state Potts model on a random planar lattice", hep-th/9502014.

[14] F. Peruggi, J. Phys. A16 (1983) L713.

F. Peruggi, F. di Liberto and G. Monroy, J. Phys. A16 (1983) 811;

F. Peruggi, Physica 141A (1987) 140;

F. Peruggi, F. di Liberto and G. Monroy, Physica 141A (1987) 151.

[15] F. Peruggi, F. di Liberto and G. Monroy, Z. Phys. B66, (1987) 379.

[16] D. Sherrington, J. Phys. A13 637 (1980);

R. Penney, A. Coolen and D. Sherrington, J. Phys. A26 3681 (1993). 


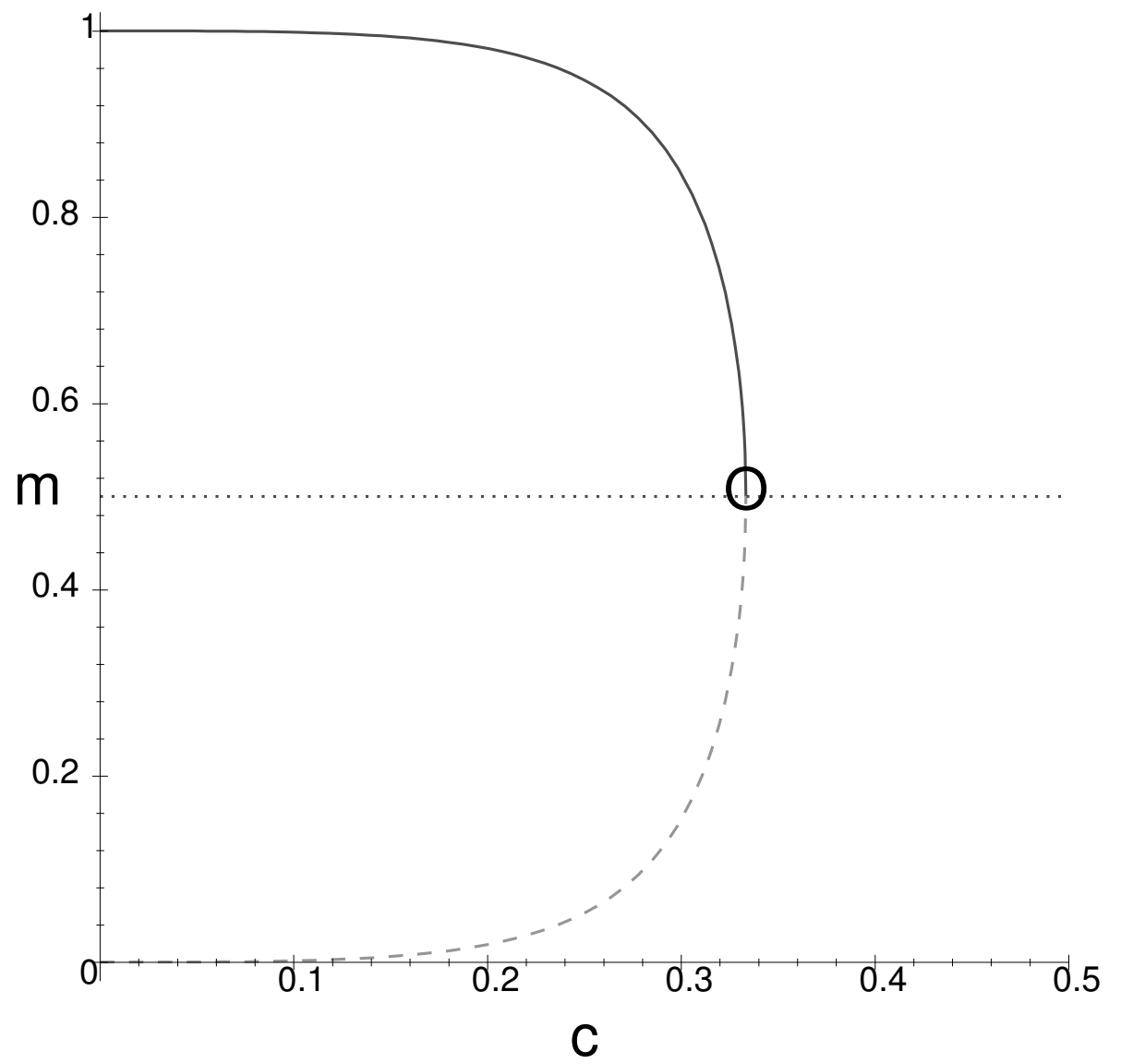

Figure 1: The magnetisation $m$ for the Ising model as calculated from the saddle point solutions. The high temperature branch is shown dotted, the upper low temperature branch solid and the lower low temperature branch dashed. 


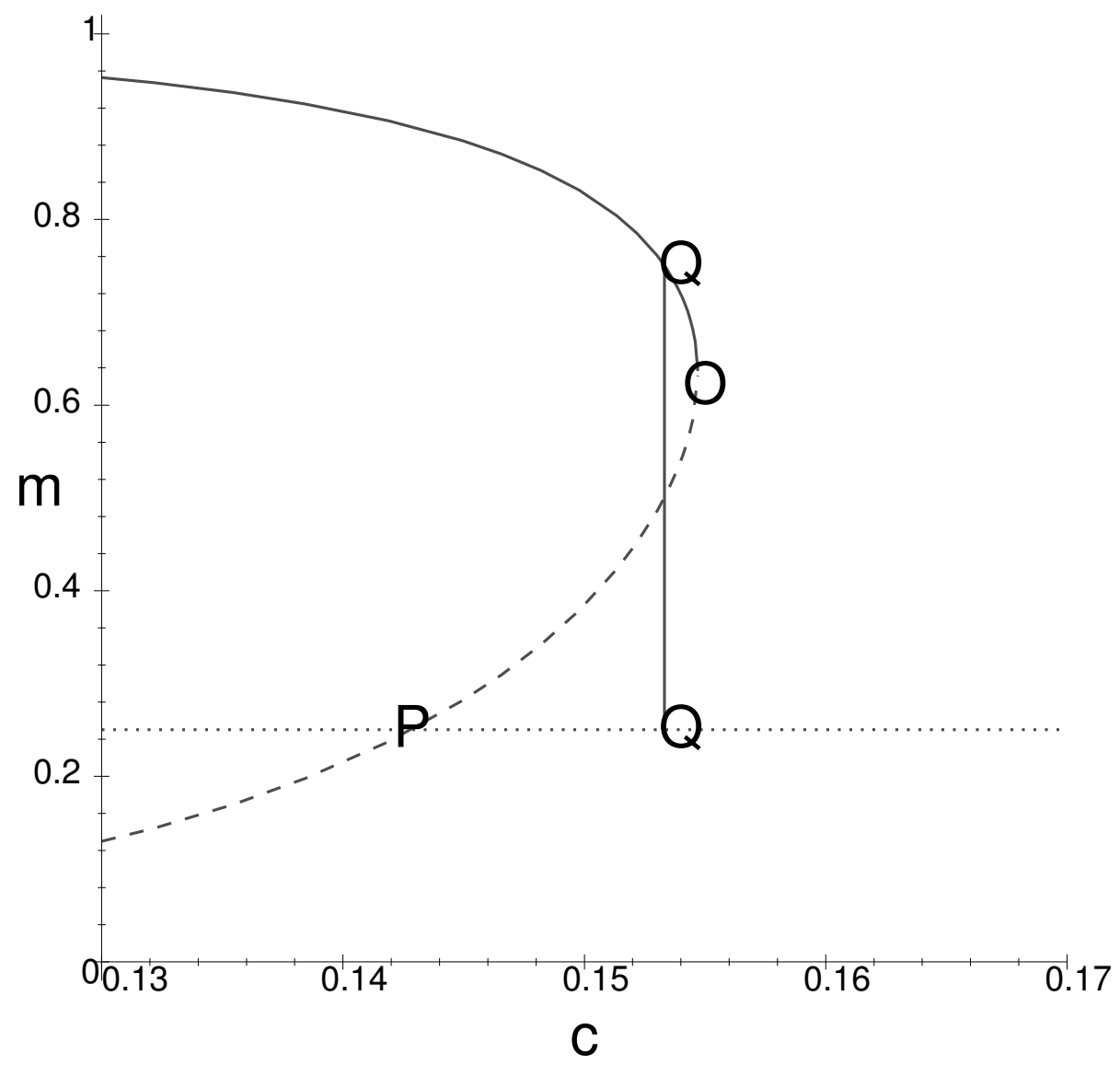

Figure 2: The magnetisation $m$ for a 4 state Potts model as calculated from the saddle point solutions. The linestyles are as in Fig.1 and only the portion of the graph close to the transition point is shown for clarity. 


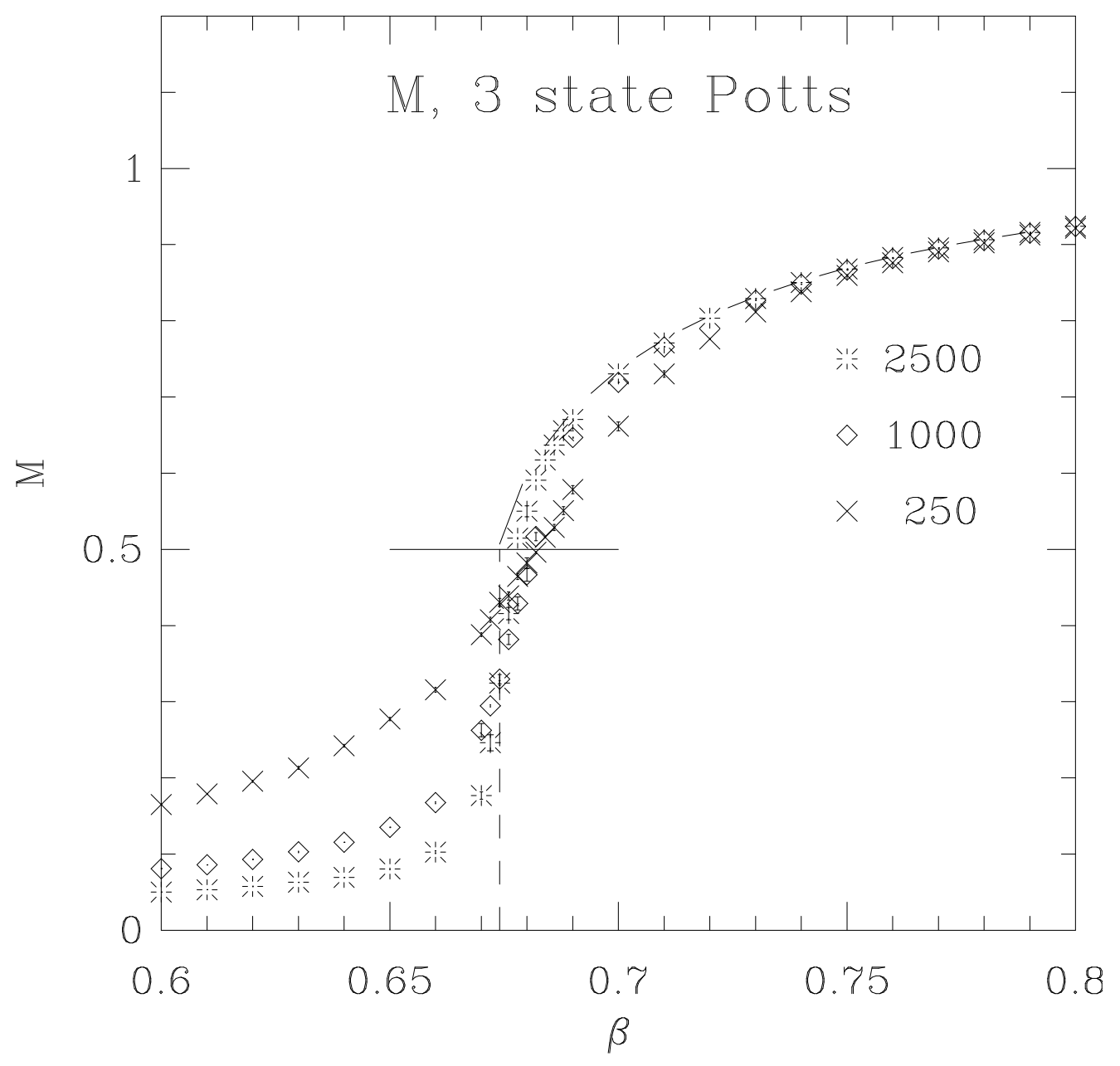

Figure 3: The magnetisation $M(=(q \max (m)-1) /(q-1))$ for a 3 state Potts model as measured in the simulations. The saddle point solution is shown as a dashed line. The horizontal bar represents the height of the "jump" $0 \rightarrow \Delta M$ in the magnetisation. The error bars are too small to be seen in all but the central points 


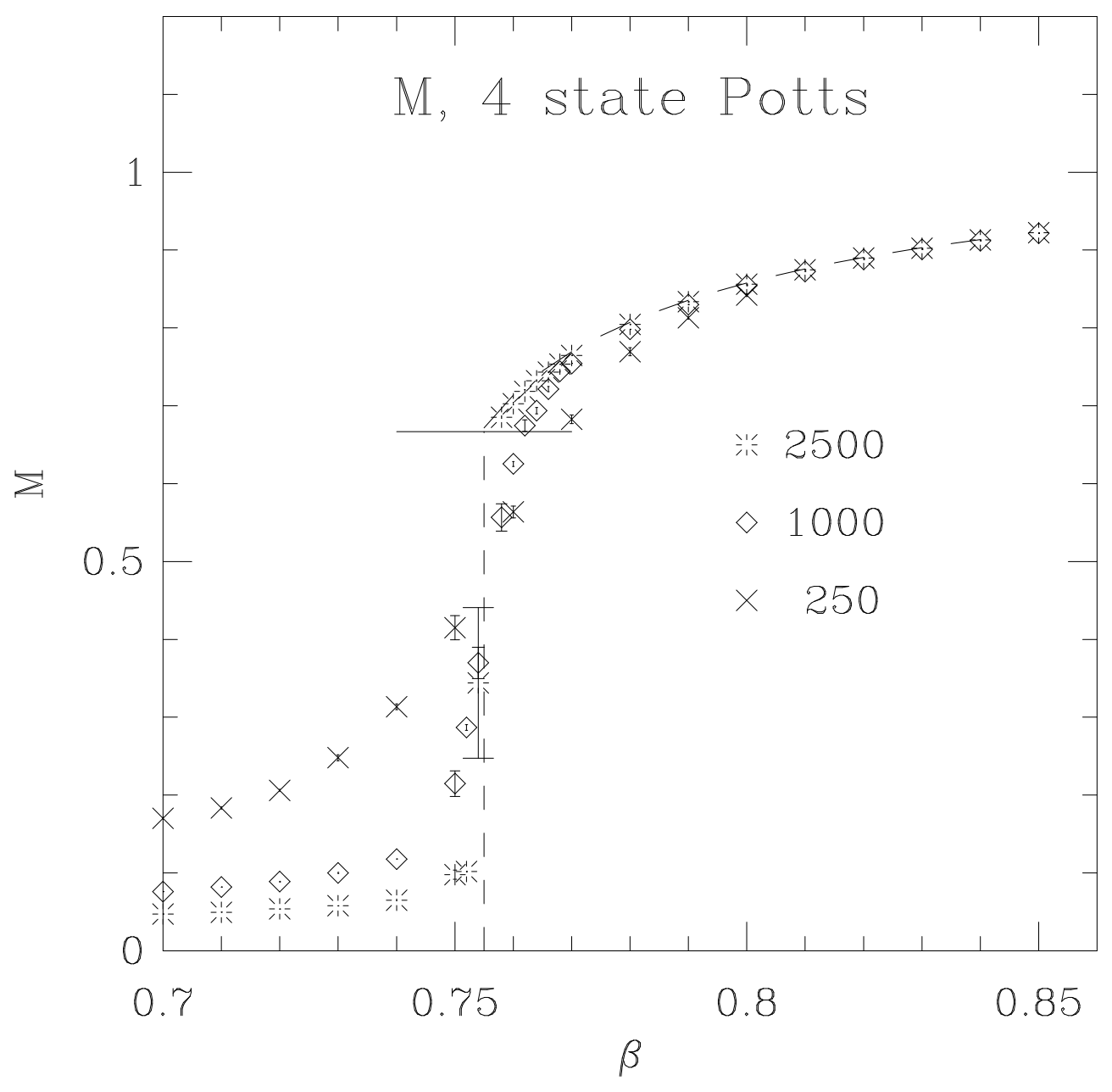

Figure 4: The magnetisation $M$ for a 4 state Potts model as measured in the simulations. Key as for Fig.3. 


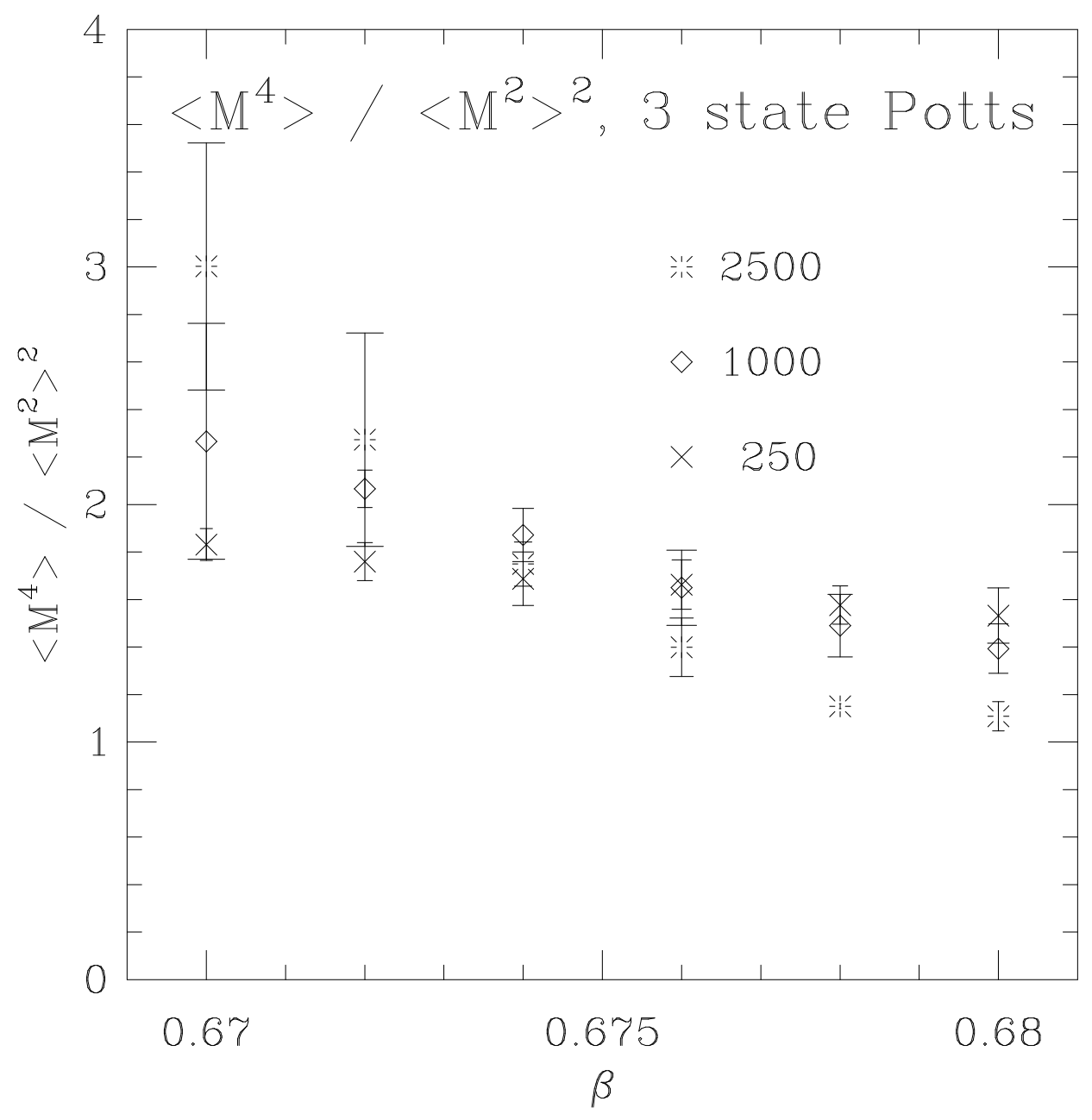

Figure 5: The crossing of Binder's magnetisation cumulant for the 3 state Potts model as measured in the simulations. 


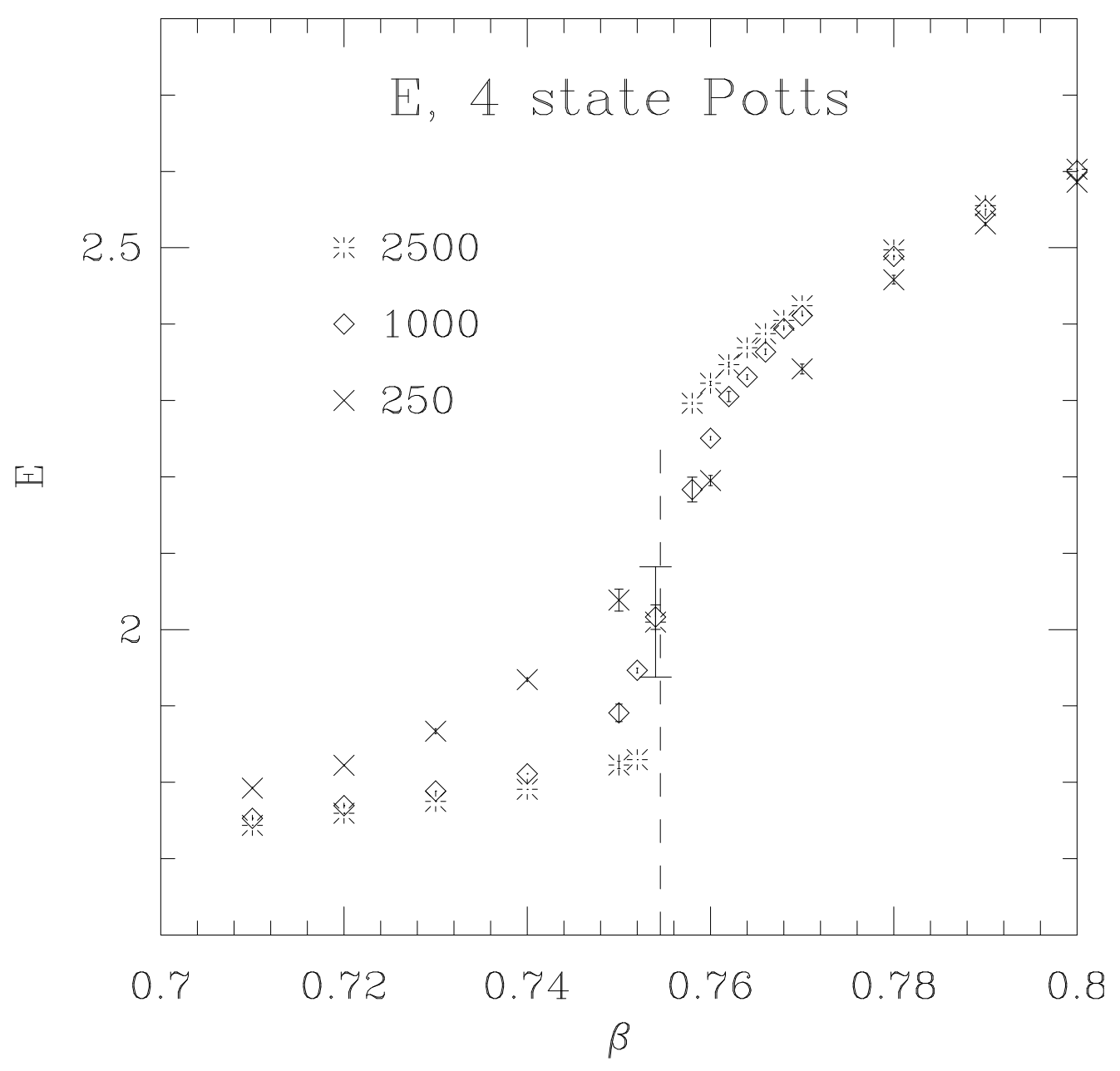

Figure 6: The energy for a 4 state Potts model as measured in the simulations. The position of the transition as calculated in the saddle point approximation is shown again as a dotted line. 